\title{
E08 STOCHASTIC OBJECT-BASED SIMULATION OF TURBIDITIC CHANNELS CONSTRAINED BY WELL, PROPORTION AND HIGH RESOLUTION SEISMIC DATA
}

Bureau F221, Bat. F, ENSG, Rue du Doyen Marcel Roubault, BP 40, 54501 Vandoeuvre-Les-Nancy, France

\begin{abstract}
The challenge of the object-based simulation approach presented in this paper is not only to account for well descriptions and proportion data but also to include channel parts inferred from seismic data analysis into the simulations. Indeed, recent progresses in exploration techniques (sonar images, high resolution seismic data and processing algorithms) enable the study of the detailed morphology of deep marine fans. In turbiditic systems, sinuous channel parts have been observed on high-resolution seismic data. Integrating such data then become essential for building realistic 3D reservoir models upon which field development strategies are based.
\end{abstract}

\section{Introduction}

Technology progresses in reservoir data extraction make the incorporation of different data sources within stochastic image procedures more and more important. Recently, thanks to high resolution seismic data interpretations, parts of fluvial bodies may be available [1,2] in addition to well and proportion data. One recent challenge in stochastic simulation realm is then to simulate the non-visible parts of these fluvial bodies, in the continuity with the observed ones.

Object-based simulation methods are special types of modeling that aim at stochastically creating and distributing idealized facies features (objects) with well-defined geometry, conditioned to available subsurface data. The resulting models have the benefit to provide a realistic representation of complex features such as channels and also, to define connectivity between the objects and continuity within each object [3]. However, object-based methods often have difficulties to satisfy hard and soft data while providing several models (or realizations) in a reasonnable amount of time.

The object-based approach described in this report aims at generating efficiently turbiditic channel stackings honoring diverse combinations of data types such as: well observations, proportion data and also parts of sinuous (meandering) channels. On one hand, well data and channel parts are taken into account using a direct (non-iterative) process. On the other hand, soft data such as proportion data are reproduced thanks to an iterative process. The efficiency of the proposed algorithm is essentially due to the so-called direct process and an optimized technique for updating the current proportion of simulated channels.

After defining the geological characteristics of the 3D models to reproduce, the object model used for modeling channels will be explained. Then, the direct process algorithm will be briefly described. Focus will be devoted to the integration of channel parts, further details about the whole algorithm are available in [4]. Finally, applications will illustrate the different abilities of the proposed approach. 


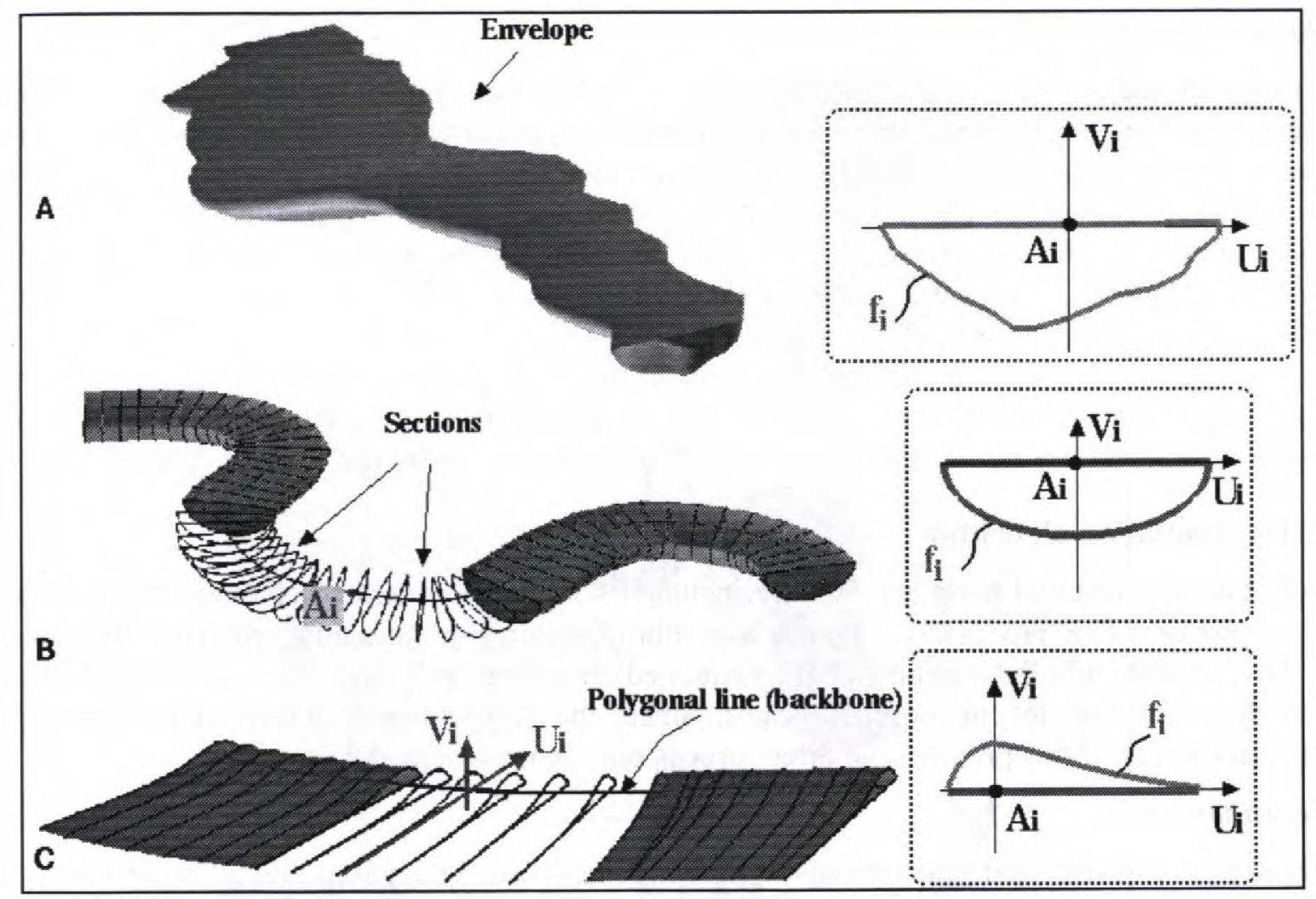

Figure 1 : Parametric shape design and three examples of modeled fluvial bodies using this data structure : A, broad channel ; B, sinuous channel ; C, levee.

\section{Geological and geometrical models}

Characterization of a fluvial reservoir generally starts with the modeling of its major structures such as layers and fault blocks. 3D curvilinear grids are discretized data structures commonly used for representing the 3D reservoir geometry. Since the grid mesh is built so that it follows the geological layering, stratigraphic coordinate transformations $\Phi$ and $\Phi^{-1}$ provide the change from, respectively, the real space (folded and faulted) to the deposition one and conversly.

Within the reconstructed $3 \mathrm{D}$ stratigraphic model, different representations of facies associations are simulated. In object-based approaches, facies repartitions are defined by the distribution of idealized elements. Thus, two essential issues are addressed below : the geological characteristics of the elements to generate and the data structure to use for modeling them.

\section{Geological framework}

Up to now, no conceptual geological model seems to be appropriate for including the diversity of actual turbidite system architectures. This leads geologists to consider [5] that one important key for studying turbiditic systems is to analyze the characteristics of the five following features : major erosion features, channels, overbank deposits, lobes and channel-lobe-transition deposits.

Moreover, since high resolution seismic data allow the extraction of fluvial body parts, they can be used as analogue data for reservoir characterization. From such turbiditic system study, it appears [1, 2] that turbiditic channel geometry (width, bend amplitude and frequency) often evolves from the bottom to the top of the canyon so that it leads to meandering channels.

For object-based modeling purpose, extracted channel parts can provide : (1) a picture of the fluvial body shapes the simulation algorithm has to reproduce; (2) data to honor, which means to simulate the non-visible parts of these channels. 


\section{Fluvial element model}

In the proposed approach, a parametric shape is used for reproducing turbiditic channel bodies. It is composed of a polygonal line, called backbone, that carries several sections. Each section consists of a vector basis defined so that the section plan is perpendicular to the local tangent of the backbone line and of a function $f_{i}$ describing the local fluvial body profile defined in the local basis (Figure 1). The section profile can either be defined by an analytical function (semi-elliptic for Figure 1-B and stemming from [6] for Figure 1-C) or by a piecewise linear function that can be simulated by a gaussian field (Figure 1-A).

Two main advantages follow from this object model : (1) it has no limitation concerning the reproduction of meandering channels; (2) as the sections are locally perpendicular to the backbone line, it does not lead to "bottlenecks" in the sinuous bend parts of the channel.

\section{Direct simulation algorithm}

Well data and channel parts are directly included within the generated channel objects using a direct process. This process is a 4-steps algorithm that aims at simulating channels that directly fit the hard data. On the contrary of the simulated annealing technique [7], it is not iterative and then does not lead to convergence issues. Since, the integration of interpreted channel parts concerns stages of this process, the direct proces outline is presented below.

\section{Algorithm outline}

Through a reservoir study, deterministic data may be available such as fluvial body boundaries along wells or on seismic horizons, correlations between wells and finally channel-belt locations. The proposed algorithm is then based on the following principle : if deterministic data are not available, they are simulated thanks to a stochastic process. The algorithm may be then summarized as follows :

- Step 1, hard data processing : pieces of channel are stochastically generated from hard data as series of cross-sections. For example, from channel facies well intervals, one or several pieces of channels (Figure 2-1) are generated in such a way that they satisfy a priori size, direction and stacking parameters.

- Step 2, channel data correlation : the different pieces of channels generated from the step 1 are stochastically correlated across the 3D space (Figure 2-2). Through this process, two objectives are investigated : (1) to decide which channel pieces are contained within the same channel according to several criteria (slope, direction or distance between data);(2) to provide a geologically consistent vertical channel stacking. At the end of this step, "chronological surfaces" are built and each of them will contain one channel top.

- Step 3, channel-belt generation : a channel-belt are simulated within each chronological surface (Figure 2-3) thanks to preferential direction law and two gaussian fields defining its sinuosity and its width. By computing intersections between the channel-belt area and the background facies well intervals, conditioning "repulsive" locations through which the channel must not pass are collected. They will be used to condition the channel middle-line simulation so that, once generated, the channel avoids the "repulsive" points.

- Step 4, channel body simulation : a channel element is generated using a set of three 1D gaussian fields (backbone, width and thickness) [7] that are conditioned by the origins of the channel cross-sections and the computed conditioning points (Figure 2-4).

As a result, several channels are simulated and satisfy by construction the hard data (for example, well intervals where channel facies is met). These channel objects also account for the input geological parameters (dimensions, slope, direction and sinuosity). 


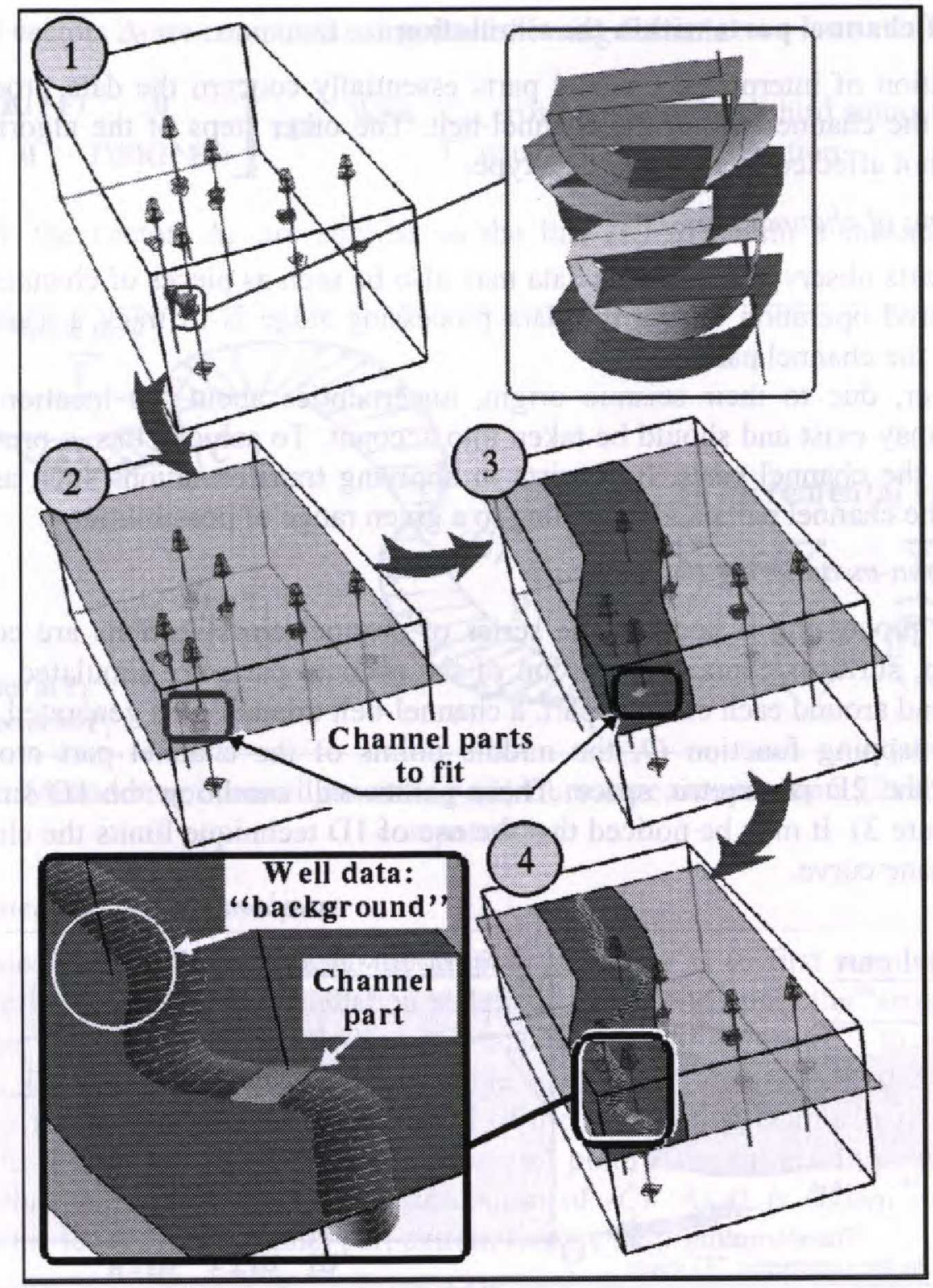

Figure 2 : Steps of the proposed direct process ( $\mathrm{Z}$ axis streched by 10$)$.

\section{Interest of using a hierarchical approach}

Hierarchical object-based approaches are interesting [6] for reproducing the primary and secondary sinuosity of the channels.

In the proposed approach, due to the use of the channel-belt area on which a mapping function $\varphi$ and a parametrization $\varphi^{-1}$ [8] are defined, the channel body simulation simply consists in generating a middle-line (backbone) in 2D and sections, whatever the complexity of the channel-belt (folded or faulted).

\section{Interest of using $1 D$ gaussian fields}

Two main benefits follow from the use of gaussian fields. It first enables the sinuosity of the simulated channel to be irregular but controled by the variogram parameters (range and sill) contrary to methods using classical sinusoidal functions. Unlike other approaches using gaussian fields [9], the simulation algorithm proposed here utilizes a second powerful advantage of 1D simulation techniques: they can be conditioned to local points through which the generated line has to pass. In this way, it is easy to enforce the channel object to pass through data points. 


\section{Integration of channel parts within the simulation}

The incorporation of interpreted channel parts essentially concern the data processing and the simulation of the channel within the channel-belt. The other steps of the algorithm previously described are not affected by this new data type.

\section{Data processing of channel parts}

The channel parts observed on seismic data may also be seen as pieces of channel objects. Then, the only required operation during the data processing stage is to track a sampling of crosssections along the channel parts.

However, due to their seismic origin, uncertainties about the location of interpreted channel parts may exist and should be taken into account. To achieve this, a pre-processing can be applied on the channel parts. It consists in applying transformations such as translation or rotation onto the channel parts and according to a given range of possibilities.

Integrating a non-meandering channel part

Once the data processing is applied, the series of channel cross-sections are correlated in the 3Dspace. Then, surfaces containing the top of the channel parts are simulated. Finally, within each of them and around each channel part, a channel-belt domain $C_{t}$ is generated.

Thanks to a mapping function $\varphi$, the middle points of the channel part cross-sections are projected into the $2 \mathrm{D}$ parametric space. These points will condition the $1 \mathrm{D}$ simulation of the backbone (Figure 3). It may be noticed that the use of 1D technique limits the channel sinuosity to be a one-to-one curve.

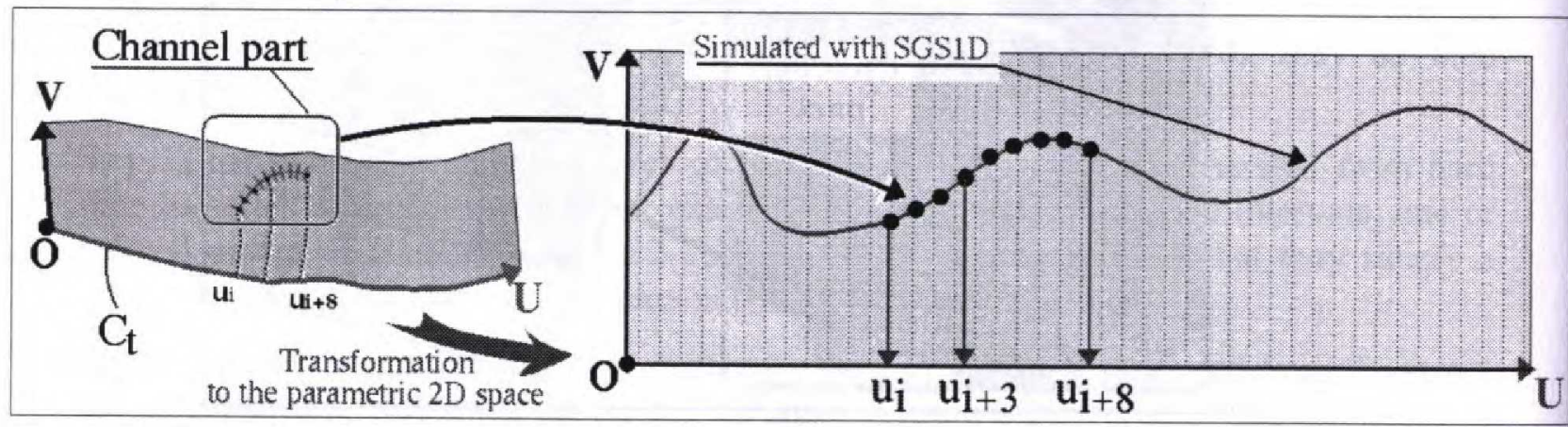

Figure 3 : Integration of a one-to-one channel part within the channel smulation.

\section{Simulating a meandering channel middle-line}

Several methods have been proposed for generating meandering channels $[10,11]$. However, these works only concern the evolution of a continental river through the time and they use hydraulic parameters that can not be available from reservoir studies. Consequently, we propose a geometrical approach for generating a meandering channel element from an arbitrary one. This approach only requires geomorphological parameters such as sinuosity and dimensions whose value ranges can be inferred from channel parts analysis. This method, denoted to "erosion process" in the following, is summarized below but further details may be found in [12].

It considers an arbitrary original channel whose polygonal middle-line is denoted to $(C)$. At each point $\mathbf{P}_{\mathrm{i}}$ of this line, a primary series of vectors $\mathbf{D}_{\mathbf{i}}$ are computed as a linear normalized combination of a given flow vector $\mathbf{F}$, the local tangent vector $\mathbf{T}_{\mathbf{i}}$ and the local curvature vector $\mathbf{K}_{\mathbf{i}}$. This vector field gives the major trend of the middle-line displacement but has to be modulated by the curvature intensity in order to reproduce meandering bends. 
Thus, the final vectors $\Delta_{\mathbf{i}}$ are computed using the following formula :

$\Delta_{\mathbf{i}}=\mathbf{C e} \cdot\left[\begin{array}{cc}\Gamma(\mathbf{K} \mathbf{i} \cdot \mathbf{F}) & \mathbf{0} \\ \mathbf{0} & \Gamma\left(\left\|\mathbf{K} \mathbf{i}^{\wedge} \mathbf{F}\right\|\right)\end{array}\right]$. Di $_{\mathbf{i}} \begin{gathered}\text { With }: \mathrm{C}_{\mathrm{e}}, \text { erosion coefficient (third sinuosity) } \\ \Gamma, \text { gaussian filtering function }\end{gathered}$

Once obtained, the vectors $\Delta_{\mathbf{i}}$ are applied on the line $(C)$ to obtain a meandering line $\left(C^{\prime}\right)$ (Figure 4).

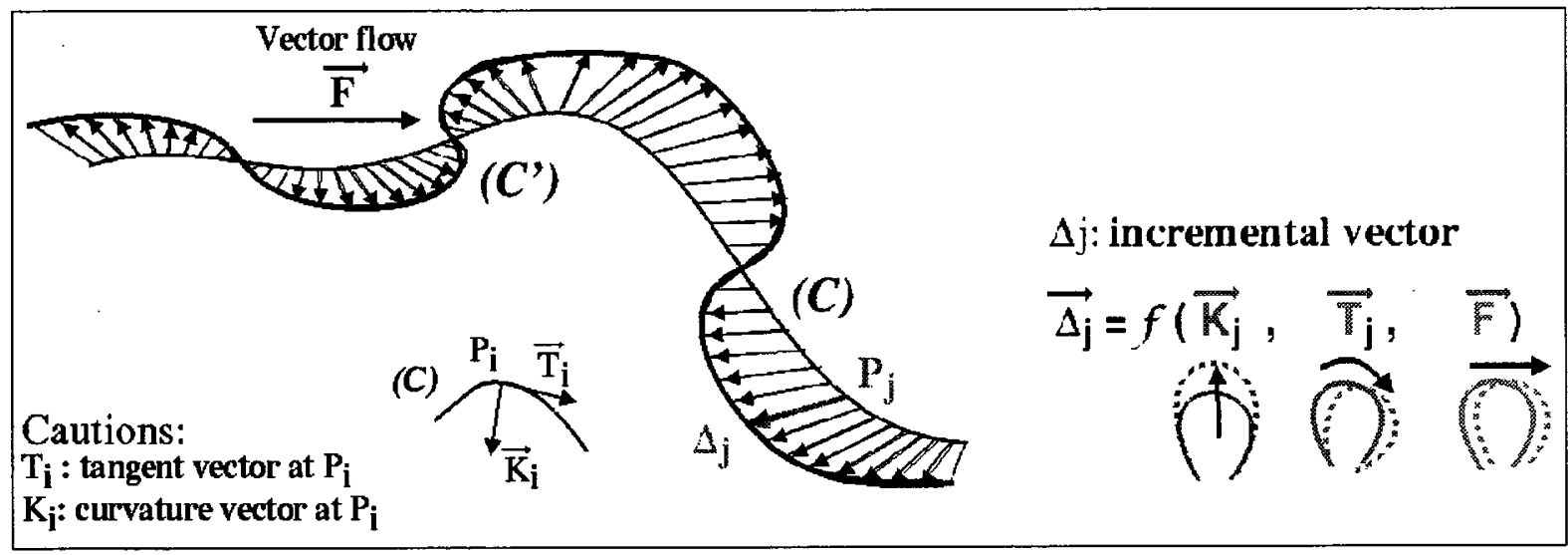

Figure 4 : Meandering channel generating ;(1) Curvature computation;(2) shift vector computations.

\section{Integrating a meandering channel part}

To integrate meandering channel parts, the proposed strategy is to first simulate a one-to-one channel middle-line $(C)$ via a $1 D$ simulation technique, to second apply the "erosion process" in order to obtain a meandering middle-line $\left(C^{\prime}\right)$ and finally, to adjust $\left(C^{\prime}\right)$ to the meandering channel parts. To minimize the deformation due to this last adjustment, the first simulated middle-line $(C)$ has to reproduce the bend trend of the meandering channel part to incorporate. To achieve this, it is proposed to compute "strategic" points (the inflection points, the maxima and minima) that will condition the ID simulation of $(C)$. As it is shown in Figure 5, the adjustments at the level of the channel part extremities are negligible.

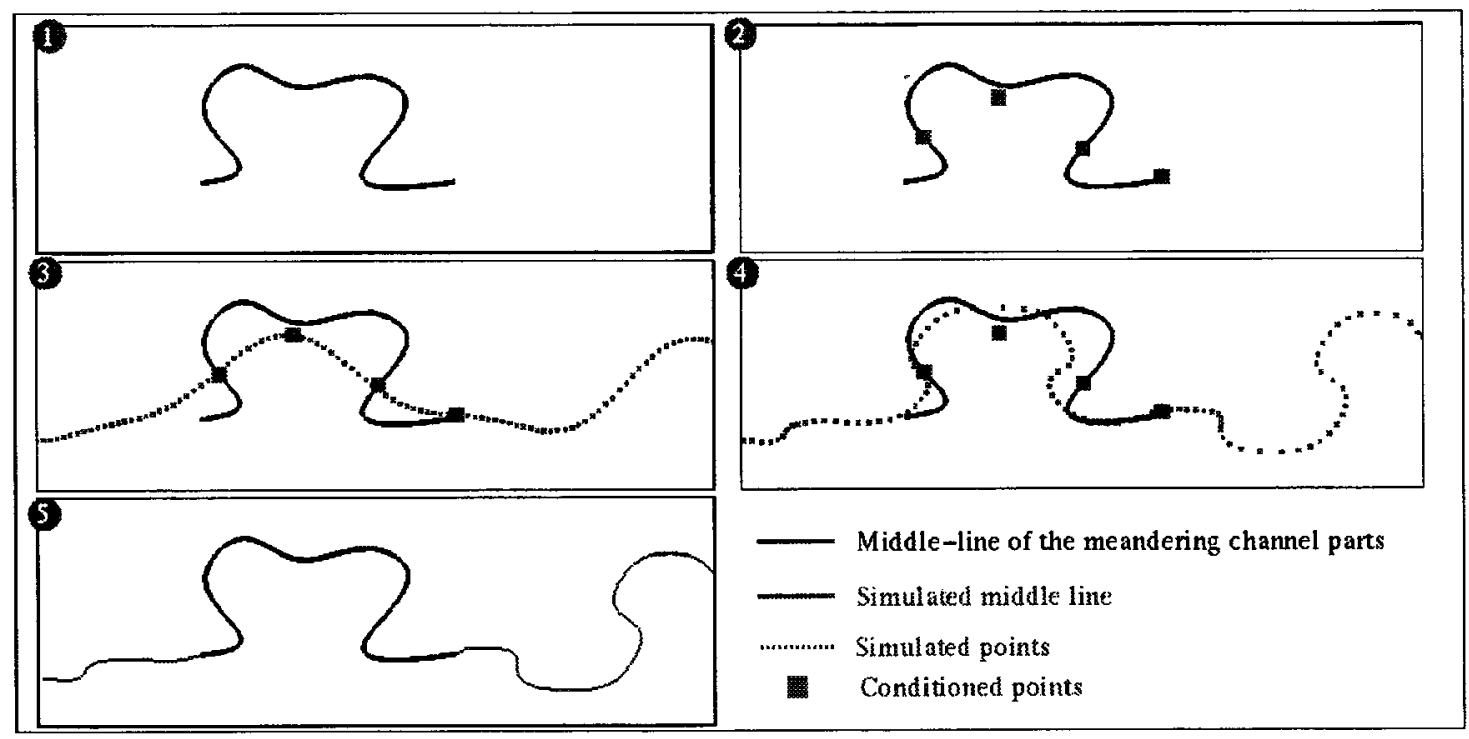

Figure 5 : The five stages of the incorporation of meandering channel parts. 
However, as the obtained channel is not defined by a one-to-one middle-line, it may intersect well intervals belonging to background facies.

\section{Integrated approach and applications}

The direct process previously described has been included in a global iterative algorithm to allow the accounting of an a priori channel facies proportion. This global procedure may be either a classical Metropolis-Hasting algorithm [7] as it is performed in [13] or a "gradient based" one as for the applications shown in this report. By using the direct process for simulating each channel objects, one channel stacking 3D model accounting for soft and hard data can be obtained in a few iterations, which enables one to provide multi-realizations in a reasonnable amount of $C P U$ time.

The speed of the presented algorithm is also due to a fast update of the proportion. It is an optimization of classical approximation techniques (called rasterizations) that is based on efficient algorithms inspired from very known techniques in computer graphic realm [14].

Different proportion trends such as vertical proportion curve and/or areal maps of proportions can be also reproduced. Vertical and areal local $c d f$ are computed thanks to, respectively, the vertical proportion curve and the areal map of proportion. Channel middle-line locations are simulated and controled via a Monte Carlo sampling [7] applied on these local cdf.

As shown in Figure 6, a synthetic dataset has been built to test the ability of the proposed approach for combining several data types. This reservoir dataset is composed of : vertical well observations, a vertical trend of facies proportions calibrated to the global proportion value, a map that defines the geometry of the channelized zone, the geological knowledge about channel geometry and four interpreted channel parts.

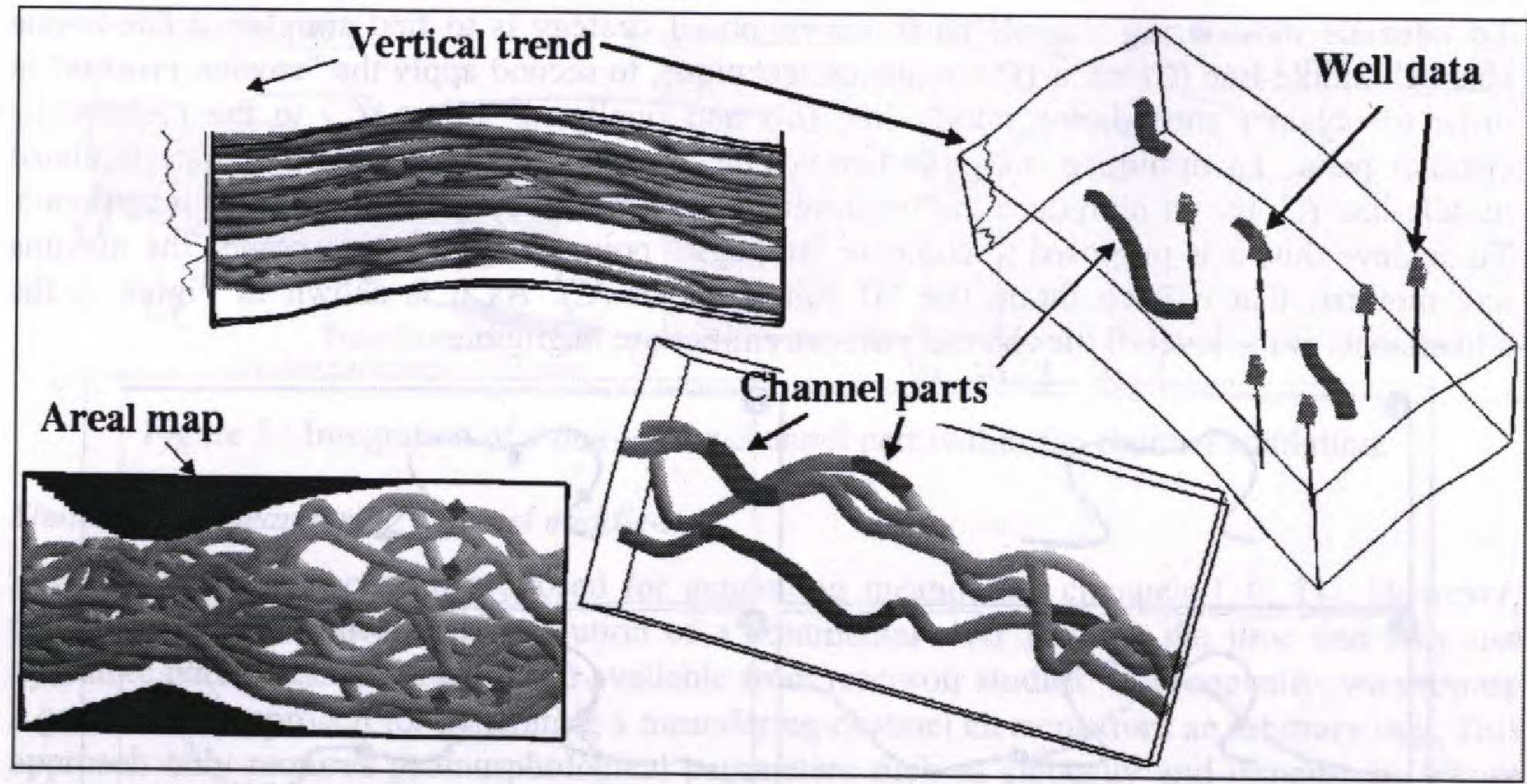

Figure 6: Results from synthetic reservoir dataset composed of: well observation, vertical proportion trend, areal trend, channel parts and geolgical knowledge ( $\mathrm{Z}$ axis stretched by 10 ). CPU time : 5 seconds on a PC Pentium III, in 4 iterations.

\section{Conclusion}

The approach described in this paper has been developed in response to the difficulty generally met in object-based approaches to simulate both efficiently and precisely channel stacking models. As the used direct process is a kind of stochastic counterpart of a deterministic analysis

$8^{\text {th }}$ European Conference on the Mathematics of Oil Recovery - Freiberg, Germany, 3 - 6 September 2002 
of reservoir data, several types of interpreted data can be easily incorporated within the whole stochastic procedure without changing its outline. In this paper, we have shown that the integration of a novel data type such as interpreted channel parts does not require dramatical changes in the algorithm implementation. However, efforts have to be devoted to assert that the generated meandering channels do not intersect the background facies intervals.

\section{Bibliography}

1) J. Peakall, W. D. McCaffrey, B. C. Kneller, C. E. Stelting, T. R. McHargue and W. J. Schweller, A Process Model for the Evolution of Submarine Fan Channels : Implications for Sedimentary Architecture, AAPG Memoir 72 / SEPM Special Publication, Vol. 68, pp. 73-99, 2000.

2) J.P. Wonham, S. Jayr, R. Mougamba and P. Chuilon, 3D Sedimentary Evolution of a Canyon Fill (Lower Miocene-age) from the Mandarove Formation, Offshore Gabon, Marine and Petroleum Geology, Vol. 17, pp. 175-197, 2000.

3) T. A. Jones and D. K. Larue., Object-Based Modelling and Deepwater Depositional System, IAMG, Proceedings of IAMG'97, CIMNE, pp. 438-443, 1997.

4) S. Viseur, A. Shtuka and J-L. Mallet, New Fast Object-Based Simulation of Fluvial Deposits, Proceedings of the SPE Annual Technical Conference, SPE number 49281, 697-709, Vol. $\Omega$, New Orleans, 1998.

5) E. Mutti and W. R. Normack, An Integrated Approach to the Study of Turbidite Systems, Seismic Facies and Sedimentary Processes of Submarine Fans and Turbidite Systems, Springer-Verlag, Ed. P. Weimer and M.H. Link, pp. 75-105, 1991.

6) C.V. Deutsch and L. Wang, Hierarchical Object-Based Stochastic Modeling of Fluvial Reservoirs, Mathematical Geology, pp. 857-880, Vol. 28, No 7, 1996.

7) P. Goovaerts, Geostatistics for Natural Resources Evaluation, Applied Geostatistics, Oxford University Press, 483 p., 1997.

8) M. S. Floater, Parametrization and Smooth Approximation of Surface Triangulations, Computer Aided Geometric Design, Vol. 14, pp. 231-25, 1997.

9) L. Holden and R. Hauge and O. Skare and A. Skorstad, Modeling of Fluvial Reservoirs with Object Models, Mathematical Geology, Vol 30, $\mathbf{n}^{\circ}$ 5, 473-496, 1998.

10) T. Sun and P. Meakin and T. Jøssang, A simulation Model for Meandering Rivers, Water Resources Research, Vol. 32, nº 9, pp. 2937-2954, 1996.

11) V. Teles and G. de Marsily and E. Perrier, A New Approach for Modelling Sediment Deposition in an Alluvial Plain in order to Display its Heterogenety, C. R. Académie des Sciences, Vol. 327, pp. 597-606, 1998.

12) S. Viseur, J-L. Mallet, Simulation de Chenaux Méandriformes sous Contraintes, 7ème Congrès de Sédimentologie, Nancy, France, p. 317-318, 1999.

13) T. Tran, Developments in Object-Based Modelling: Object Parametrization and Data Conditioning, EAEGE-SPE, International Symposium on Petroleum Geoastatistics, \#21, 1999.

14) J.D. Foley and A. Van Dam, Fundamentals of Interactive Computer Graphics, The Systems Programming Series, Addison-Wesley Publishing Company, 1983. 